\title{
Sic MOSFET Modeling and Simulation for Pspice
}

\author{
Xiafei Zhu, Guolin Xu, Shaokang Jiao, Zhibin Zhao
}

Department of Electric Engineering, North China Electric Power University, Beijing, 100000, China

Keywords: Sic MOSFET, circuit model, Pspice.

\begin{abstract}
Sic (silicon carbide, Sic), represented by the third generation of semiconductor devices with its superior performance, has obvious advantages on the voltage level, working temperature, switching losses. Then the size, weight, cost of the power electronic conversion may be significantly decreased and making the performance of power electronic systems improved comprehensively. Sic MOSFET has aroused special attention, which shows a tremendous potential on high voltage, high power, high temperature applications. With the deepening of the research, building accurate Sic MOSFET device model is the key to power electronic circuit simulation. Accurate modeling is even more significant to help researchers know more information of device performance and do further design to take advantages of Sic MOSFET. In this paper, a simple PSpice model for Sic MOSFET, CMF20120 is presented, based on the large number of existing models of the power MOSFET discrete devices, according to CMF20120 library files provided by CREE incorporated company. As the comparisons among standard datasheet, the simulation results based on the proposed model match very well.
\end{abstract}

\section{Introduction}

With the continuous development of material science and technology, silicon carbide as a new wide band gap semiconductor materials [1] has high breakdown field, high saturated electron drift velocity, high thermal conductivity, high melting point, low on-state resistance and other excellent physical and electrical properties, which are targeted by the experts in the field of semiconductor. [2]

Due to the unique physical structure of silicon carbide, the power semiconductor devices based silicon carbide material have a significant advantage in high voltage, high temperature and high power applications [3]. Sic MOSFET compared with the same power devices based silicon material, has a lower on-state resistance and switching losses, a wider range of operating temperatures, making the stability improved greatly. These excellent performances break the limitations of Si MOSFET due to the physical characteristics of the material. Sic MOSFET application areas covers aviation, aerospace, military, oil exploration, telecommunications, electricity, etc. [4], so the Sic MOSFET device research has become a hot topic in the scientific community.

At present, the application of silicon MOSFET in modern electronic system is already quite mature. While the high voltage, high-temperature, high-speed switching characteristics, low loss, high stability characteristics of Sic MOSFET making it more consistent with the need for the development of power semiconductor devices, has become the focus of the power device research. Meanwhile circuit simulation on Sic MOSFET devices are more and more important. The modeling of power semiconductor devices is a key power electronic circuit simulation, accurate circuit model can be more accurate, analogy, normal, fault operating, and it is of great significance for the safe and stable operation of the system. Therefore it is necessary to establish an accurate Sic MOSFET equivalent circuit model for circuit simulation.

\section{The all equivalent circuit model}

In recent years, equivalent circuit model of Sic MOSFET has been studied by domestic and foreign researchers, and got remarkable achievements. Traditional power device modeling divides into physical models and behavior models. The physical model is a solid state physics knowledge-based model, solving the semiconductor device equations. This method can accurately describe the physical characteristics of the device, and it is suitable for process manufacturing simulation 
applications. A good physical model of power devices can be built using the TCAD system software, [5] build a physical model of the Sic MOSFET by using the ATHENA and ATLAS software and however the model will take a lot of time and memory space, and is not suitable for power system simulation analysis applications. Reference [6] propose a mathematical-physical model which has uniform parameters which can describe the static and dynamic characteristics of Sic MOSFET device accurately, but this model is too complex for complex circuit simulation applications. Currently, behavior modeling is most popular, the model is built based on the external characteristics of devices. The parameters of the model are easy to obtain as previously described, the model can be satisfied with the needs of the specific circuit simulation, but compared with physical model it is less accurate. Spice software provides a typical MOSFET equivalent circuit model, as shown in Fig.1 (a). Reference [7] improved the typical MOSFET SPICE model according to the Sic MOSFET device characteristics, adding the temperature dependent voltage source and current source to compensate for static characteristic of Sic MOSFET, adding nonlinear capacitance between the gate and drain to describe the dynamic characteristics of Sic MOSFET. Reference [8] reuses the available built-in MOSFET models of the regular lateral MOSFET devices and propose a new model to depict the same behavior as expected by Sic device. The model is universal significant for Sic MOSFET modeling, and has been affirmed by researchers. Improved model shown in Fig.1 (b). Reference [9-11] optimize the key parameters of the temperature dependent voltage source and current source of the model proposed by [7]. The new model is good for low temperature analysis to $-25^{\circ} \mathrm{C}$, which can operate for a wider temperature range. However the model proposed by [10] only makes improvements to the external compensation device, no change to the core of the device. And there is little information available in literature about the core of Sic MOSFET. The model is proposed by Kai Sun presented in Fig.2.

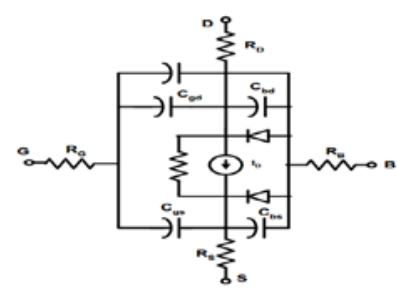

(a)

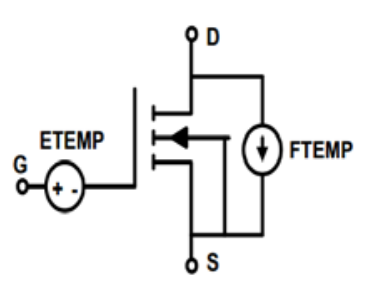

(b)

Fig.1: (a) Level 1 MOSFET Model; ～(b) Improved MOSFET Model in SPICE Software

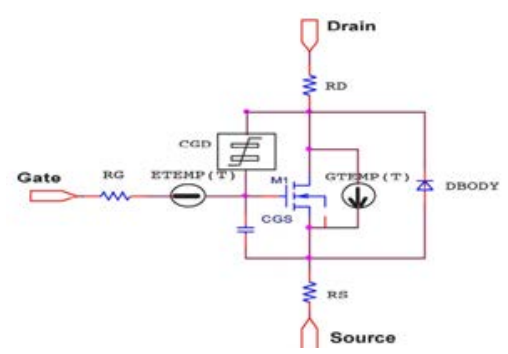

Fig.2: The model proposed by Kai Sun

This paper proposes a simplified equivalent circuit model of Sic MOSFET, CMF20120 (1200V class) which is based on the library provided by CREE, after the extensive research and analysis on the behaviour and physical model, the equivalent circuit model is built in PSpice software. This study focuses on the core part of CMF20120 SPICE library file, simplifies the temperature dependent resistance and anti-parallel diode between drain and source, extends the model's work temperature range and makes the model more accurate for describing the static characteristics of the device at different temperatures. A series of tests for CMF20120 are tested based on IEC60747 and GB/T 29332-2012 using the Agilent B1505A Power Device Analyser and Curve Tracers are not described here, as restriction of paper length. The model parameters are changed in order to make the model better suited to the tests needs, run simulations under the same conditions, and make 
comparisons among tests, simulations and standard datasheet for the static characteristics to verify the accuracy of the model. Fig.3 shows the equivalent circuit model built in the PSpice software. In this part, the detailed process modeling will be discussed. In Fig.3, gmos is the core part of the model that is used for controlling the drain current; Rdrain is a temperature dependent resistance which is use for describing the temperature effect of the whole model; body diode is the antiparallel diode between drain and source which is use for protecting the whole circuit.

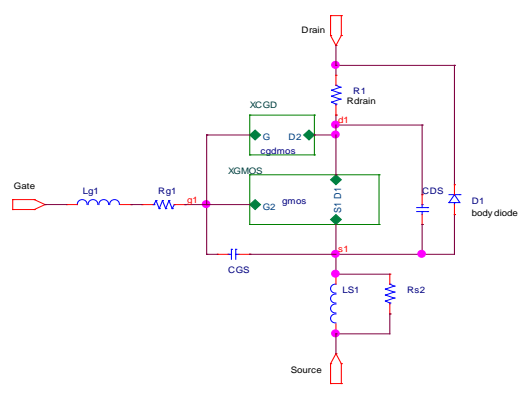

Fig.3: The Sic MOSFET equivalent circuit model

In the PSpice software, the DC Analysis with the parameter based sweep analysis is used to validate the proposed model. The DC characterization includes the output characteristic measurement, transfer characteristic measurement, etc. Fig.4 show the drain current ID as a function of drain voltage source VDS with a gate voltage source VGS as obtained from the datasheet and that obtained as parameter using PSpice model simulation.

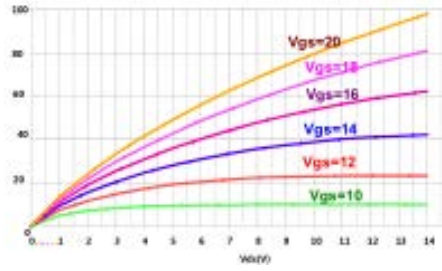

(a)

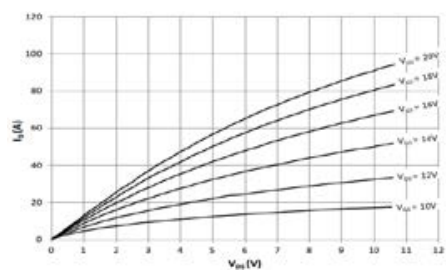

(b)

Fig.4: (a) The simulation output characteristic curve; (b) The output characteristic of CMF20120D Datasheet $=25^{\circ} \mathrm{C}$

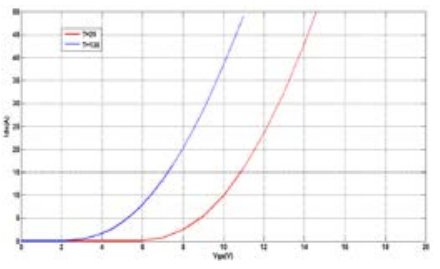

(a)

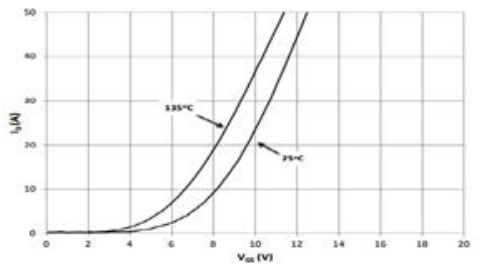

(b)

Fig.5: (a) The simulation transfer characteristic curve; (b) The transfer characteristic of CMF20120D Datasheet

The transfer characteristic of CMF20120 Sic MOSFET which is a plot of ID versus VGS at constant VDS is shown Fig.5. Fig.4 (a) and 5(a) are the simulation results, Fig.4 (b) and 5(b) are the characteristics curves of CMF20120 provided by CREE Company. From the comparison, the results show appreciable closeness with the datasheet.

The comparison of simulation results with CMF20120 datasheet shows the static characteristic curves of the proposed model in this article are similar thus justifies the accuracy of the proposed model.

\section{Core Part}

The core part is shown in the Fig.6 (a). The gmos sub-circuits has three external ports defined as D1, G2 and S1 respectively. And there are other elements such as fixed value resistors, controlled voltage source and current source. All the elements are used in presenting the CMF20120 library 
file in PSpice software. Port D1 and G2 are carried out the DC sweep analysis at the same time, and the output current through D1, S1 is observed the result shown in Fig.6 (b).

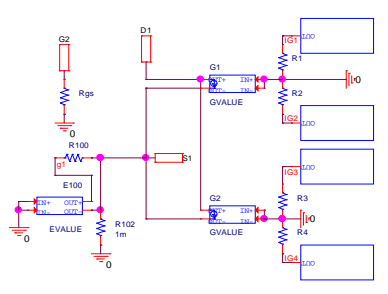

(a)

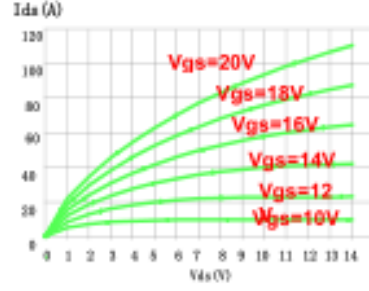

(b)

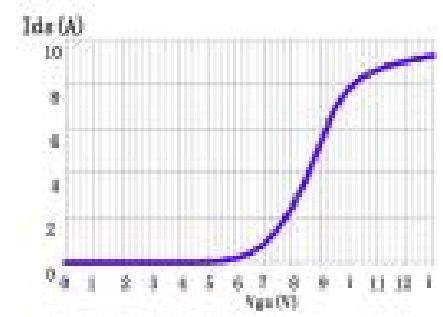

(c)

Fig.6: (a) The core part of equivalent circuit model (gmos); (b) The simulation output characteristic curve of gmos, $\mathrm{T}=25^{\circ} \mathrm{C}$; (c) The simulation transfer characteristic of gmos, $\mathrm{T}=25^{\circ} \mathrm{C}$

It can be seen that the current through D1, S1 matches the MOSFET typical output characteristic curve well. Port G2 are carried out the DC sweep analysis, and the output current through D1, S1 is observed, the result shown in Fig.6(c). It can be seen that the current through D1, S1 matches the MOSFET typical transfer characteristic curve well. From the above analysis it can be considered that the module gmos can be regarded as the kernel of MOSFET device.

\section{Temperature dependent resistance}

According to the library provided by CREE, the temperature dependent resistance model is built by using the Break component in PSpice software. The temperature dependent resistance defined by the library that is provided by CREE Company is presented as follow;

Rdrain d d1 \{(0.08+0.01)/af1 $\}$ tc1=0.000835209 tc2=2.94329E-05

The default resistance model in PSpice software is defined by (1),

$$
R=R 0^{*}\left(1+t_{c 1}{ }^{*} d t{ }^{+} t_{C 2}{ }^{*} d t{ }^{* * 2}+t_{C 3} * d t{ }^{* * 3}+\cdots\right)
$$

Where R0 is the resistance at the nominal temperature and DT is the difference between the resistor temperature and the nominal temperature.

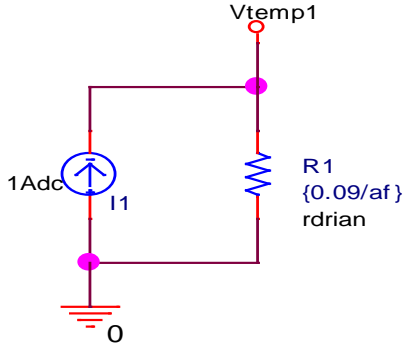

(a)

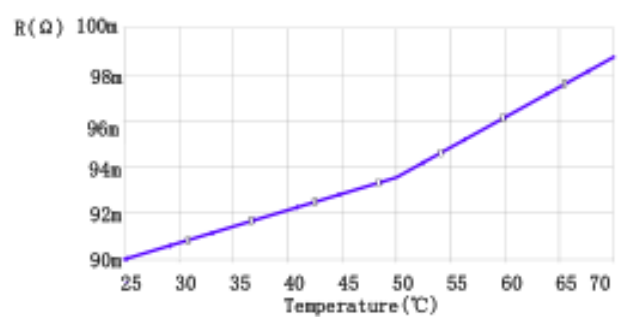

(b)

Fig.7: (a) The temperature dependent curve resistance simulation circuit; (b) Resistance versus temperature curve

Fig.7 (a) is the temperature dependent resistance simulation circuit. Fig.7 (b) shows the simulation result of temperature dependent resistance, as can be seen, the value of the temperature dependent resistance increases as the temperature increases and it is consistent with the CMF20120 datasheet. It can be considered that the temperature dependent resistance can be used for 
compensating the effect of the changing temperature. The changing of parameters in the temperature dependent resistance can affect the external feature of the MOSFET device.

\section{Body Diode}

The following is the definition of the body diode in the library provided by CREE.

Model body diode $\mathrm{d}$ (Is=1e-42 Cjo=783.3p Bv=1500 M=0.676 EG=3.26 VJ =3.82 N=1.1 $\mathrm{Rs}=0.12 \mathrm{Tt}=15 \mathrm{n}$ Ibv=1e-25 level=1)

Where:
Is_-Saturation current;
Cjo__Zero-bias junction capacitance;
$\mathrm{Bv} —$ Reverse breakdown voltage; $\mathrm{M} —$ Grading coefficient;
EG_-Activation energy; $\quad \mathrm{Vj}$ _-Junction potential;
$\mathrm{N}$ _-Emission coefficient; $\quad$ Rs_-Ohmic resistance;
Tt_-Transit-time; $\quad$ Ibv_—Current at breakdown voltage;

In this study, the body diode model is built utilizing the break element in PSpice software. And then the sinusoidal voltage source is applied to the body diode anode. From the simulation result it can be found that the output voltage of body diode cathode is similar with the typical diode. As shown in the Fig.8, it can be seen that the Sic MOSFET equivalent circuit model proposed in this study can describe the general MOSFET static characteristic adequately and the curves of output and transfer characteristic agree with the curves in CMF20120 datasheet.

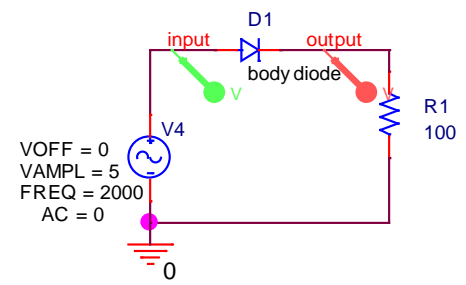

(a)

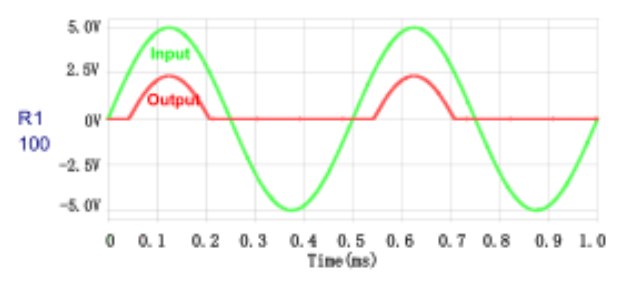

(b)

Fig.8: (a) The simulation circuit of body diode; (b) the simulation result of body diode

\section{Conclusions}

A PSpice model of Sic MOSFET is proposed, which is good for describing the static characteristic of Sic MOSFET, and is based on the library provided by manufacturer. Compared with [9]-[11],the proposed model in this study did not use the method which reuses the available built-in MOSFET models of the regular lateral MOSFET devices and propose a new model to depict the same behavior as expected by Sic device. On the contrary, in this study the modeling process of gmos is discussed in detail .The gmos can be seen as the core part like M1 in [11], which can adequately describe the static characteristics of typical MOSFET. So the external features of Sic MOSFET can be changed by gmos not only by compensational devices. It will be a new way to build the Sic MOSFET model. The model must be more accurate. However, in this paper only the static characteristics of experimental tests are carried out. The dynamic characteristics of the model will be verified and the effect from all the model parameters will be discussed in details in the future work. Then the model will be more flexible and more suitable for the circuit simulation. 


\section{References}

[1] Elasser A, Chow T P. Silicon carbide benefits and advantages for power electronics circuits and systems [J]. Proceedings of the IEEE, 2002, 90(6): 969-986.

[2] Friedrichs P, Rupp R. Silicon carbide power devices-current developments and potential applications[C]// Power electronics and applications, 2005 European Conference on. IEEE, 2005: 11 p.-P. 11.

[3] Baliga B J. The future of power semiconductor device technology[J]. Proceedings of the IEEE, 2001, 89(6): 822-832.

[4] Wang J, Yang L, Zhao T, et al. Characteristics of $10 \mathrm{kV} \mathrm{SiC} \mathrm{MOSFET} \mathrm{and} \mathrm{PIN} \mathrm{diode} \mathrm{and} \mathrm{their}$ application prospect in high voltage high frequency DC/DC converter[C]//Power Electronics Specialists Conference, 2007. PESC 2007. IEEE. IEEE, 2007: 72-77.

[5] Yunbing Gao. Sic VDMOS Device Design and Research of Interface trap effects. MS thesis. University of Electronic Science and Technology, 2013.

[6] Ramovic R, Jevtic M, Hadzi-Vukovic J, et al. A novel analytical model of a Sic MOSFET[C] //Microelectronics, 2002. MIEL 2002. 23rd International Conference on. IEEE, 2002, 2: 447-450.

[7] Wang J, Zhao T, Li J, et al. Characterization, modeling, and application of 10-kV Sic MOSFET[J]. Electron Devices, IEEE Transactions on, 2008, 55(8): 1798-1806.

[8] Pratap R, Singh R K, Agarwal V. SPICE model development for Sic power MOSFET[C]/Power Electronics, Drives and Energy Systems (PEDES), 2012 IEEE International Conference on. IEEE, 2012: $1-5$.

[9] Cui Y, Chinthavali M, Tolbert L M. Temperature dependent Pspice model of silicon carbide power MOSFET[C]//Applied Power Electronics Conference and Exposition (APEC), 2012 TwentySeventh Annual IEEE. IEEE, 2012: 1698-1704.

[10] Sun K, Wu H, Lu J, et al. Improved Modeling of Medium Voltage Sic MOSFET Within Wide Temperature Range [J]. IEEE TRANSACTIONS ON POWER ELECTRONICS, 2014, 29(5): 22292237.

[11] Lu J, Sun K, Wu H, et al. Modeling of Sic MOSFET with temperature dependent parameters and its applications[C]//Applied Power Electronics Conference and Exposition (APEC), 2013 Twenty-Eighth Annual IEEE. IEEE, 2013: 540-544. 